\title{
EL MITO DE LOS 400 MIL ABORTOS EN COLOMBIA
}

Mauricio Rubio*

$\mathrm{U}$ $n$ buen cuento ayuda a entender enigmas reales cuyo origen no se conoce pero que perduran pese al escrutinio y la crítica. La historia de Gabriel García Márquez que empieza con el augurio de una mujer que se convierte en profecía reforzada por quienes la oyen, hasta que al fin se cumple, se asemeja al conteo de abortos clandestinos. Hace décadas alguien inventó un número inverosímil de abortos en el mundo, e igual que en el cuento, sin evidencia a favor y varios indicios en contra, la cifra fue repetida y certificada por expertos, periodistas y activistas que la convirtieron en verdad inmutable, tan arraigada que todo esfuerzo para estimar un número que nadie conoce con certeza es hoy un mero trámite ritual que la confirma.

Las sospechas sobre la autoría del entuerto inicial recaen sobre la Organización Mundial de la Salud (OMS). La burocracia internacional calcula el agregado de muchas incógnitas, aun de aquellas que no se pueden estimar por separado para un país o ciudad. Sin hacer explícitas las enormes dificultades de esa tarea, calcula para el planeta magnitudes que nadie conoce para un barrio o un hogar. A una persona medianamente informada, curiosa y observadora le es difícil saber con certeza qué ocurre en las "ollas"-ventas de droga al menudeo- o en los burdeles camuflados de su vecindario. A duras penas sabe cuántos puntos de venta operan, y el cálculo de su actividad comercial es

\footnotetext{
* Docente investigador, Facultad de Economía, Universidad Externado de Colombia, Bogotá, Colombia, [mauriciorubiop@gmail.com]. Agradezco la colaboración de Santiago Plata y los comentarios de Carmen Elisa Flórez, Cristina Rosero, Alejandra Coll, Mauricio Pérez, Alberto Supelano y los asistentes al Seminario de Investigación de la Facultad. Fecha de recepción: 23 de septiembre de 2015, fecha de modificación: 20 de octubre de 2015, fecha de aceptación: 29 de octubre de 2015. Sugerencia de citación: Rubio, M. "El mito de los 400 mil abortos en Colombia”, Revista de Economía Institucional 17, 33, 2015, pp. 253-274. DOI: http://dx.doi.org/10.18601/01245996.v17n33.11
} 
pura especulación. A pesar de esa incertidumbre insuperable, para la unidad básica de observación, los burócratas internacionales y algunos académicos no reparan en calcular -con supuestos rara vez explícitos y métodos imposibles de replicar- cifras de la venta mundial de drogas, del número de prostitutas en el mundo, del dinero que reciben e incluso de las que fueron forzadas a ejercer ese oficio.

Esto también sucede con la cifra de abortos clandestinos. Son muchos los testimonios de mujeres que abortan sin contarle a su pareja, a su familia o a sus amistades.

Yo tenía una amiga a la que le contaba todo pero de esto no. Yo no podía comentarlo con nadie [...] Yo nunca había hablado de esto, nunca, nunca, ni capaz de contárselo al médico [...] nunca, jamás, en mi historia clínica jamás aparece (cit. en Zamudio et al., 1999, 93 y 135).

El silencio y el afán por no dejar huella de un acto ilegal también se observa entre quienes interrumpen el embarazo: su principal preocupación es no dejar trazas.

Salvo las personas detenidas o las pruebas recaudadas en los pocos allanamientos que hace la policía o en las aún más raras ocasiones en que los abortos clandestinos terminan con la muerte de la mujer, no queda rastro ni vestigio de ellos. A finales de los noventa, antes de hacer una encuesta en tres barrios bogotanos en los que se observaba una alta mortalidad materna, se comentó: "tener cifras específicas de cuántos se practican en Bogotá resulta casi imposible [...] no se sabe qué tipo de aborto es provocado o no $[\ldots][$ los datos] no son confiables por tratarse de un práctica ilegal" (ET, 1998a).

A pesar de esos impedimentos insalvables para saber qué ocurre a nivel desagregado, a finales de los ochenta la OMS empezó a circular una cifra de millones de abortos clandestinos en el planeta. "De acuerdo con un informe de 1987, en el mundo se practican cada año entre 40 y 60 millones de abortos" (Ladipo, 1990, 20). Este cálculo olímpico se emprendió a pesar de que años antes un grupo científico convocado por la misma OMS recomendó no hacerlo, ni siquiera para un país, dadas las dificultades de información características. Otro informe técnico de la OMS reconoció en 1992: "es casi imposible estimar con precisión las tasas de aborto" (WHO, 1992, 6).

No es coincidencia que justo después de divulgar esta cifra millonaria aparecieran en los medios colombianos datos igualmente aventurados: "Cada año se practican más de 250 mil abortos en madres menores de 19 años", declaró el ministro de salud en la apertura de un seminario sobre el tema $(E T, 1992)$. Meses después, el número fue redondeado a 300 mil por un joven periodista (Santos, 1993). Al cabo 
de un año, esa cifra fue refrendada dando a entender que provenía de una investigación rigurosa $(E T, 1994 b)$. En esa misma época, el Guttmacher Institute (GI) de Nueva York calculó que "por cada diez niños que nacen vivos en Colombia, cuatro han sido abortados" (ibíd.). El país escuchó que "Latinoamérica aborta por montones" y que la transición demográfica se estaba logrando "gracias al aborto inducido". Para esa sorprendente sugerencia se citaron cifras contundentes: "casi tres millones de abortos se provocan todos los años en cinco países"1.

En 1996 la cifra mundial fue refrendada por Naciones Unidas (Reuter, 1996), y el tema pronto dejó de ser exclusivo de la OMS. "Según datos del Fondo de Población de las Naciones Unidas, anualmente por lo menos 20 millones de mujeres se someten a abortos en malas condiciones" (ET, 1996b). La Iglesia redondeó el número de abortos eliminando la distinción entre legales y clandestinos: todos eran condenables ${ }^{2}$.

A raíz del debate en el Senado sobre la reforma al código penal, en 1998 un asesor de Profamilia retomó la cifra mundial y endosó la cifra local $(E T, 1998 b)$, a pesar de que esa entidad ya había hecho tres encuestas en el país, con una muestra representativa, que desafiaban esa cifra. En 2000, luego de que la Sala Penal del Tribunal Superior de Bogotá emitió la "primera condena por aborto", la cifra se ajustó de nuevo hacia arriba: 350 mil abortos (ET, 2000a). Un mes después, la conocida periodista Gloria Moanack, en un balance del conocimiento sobre esa práctica, señaló las dificultades para establecer su magnitud y recordó la confusión entre aborto espontáneo e inducido, pero dejó abierta la posibilidad de que este último fuera muy superior (Moanack, 2000).

Mónica Roa publicó en 2005 un excelente artículo sobre una investigación de la Universidad Externado de Colombia acerca del aborto inducido ${ }^{3}$, con una perla que reflejaba fe ciega en la cifra: si había 1,5 millones de embarazos al año y 1,1 millones de nacimientos, la diferencia "no se puede explicar de otra manera"; hay 400 mil abortos. Cuando se quiere confirmar un prejuicio, ignorar las pérdidas espontáneas es un desliz menor (Roa, 2005).

En 2011, el GI, contradiciendo sus trabajos anteriores, con una metodología y unos supuestos desfasados, "precisó" que los abortos clandestinos eran 398.700. Recientemente, de donde menos se es-

${ }^{1}$ Colombia, Chile, México, República Dominicana y Perú (ET, 1994a).

2 "Cada año se practican en el mundo unos 50 millones de abortos, aseguró ayer en Roma el cardenal Alfonso López Trujillo, presidente del Pontificio Consejo para la Familia" (Efe, 1996).

${ }^{3}$ Zamudio et al. (1999), cuyos principales resultados se resumen más adelante. 
peraba llegó también una adhesión a esa cifra. Profamilia, la entidad que hace cada cinco años la encuesta nacional de demografía y salud (ENDS), un trabajo de campo impecable con muestras representativas, endosó el mito en una campaña publicitaria, a pesar de que su encuesta -aplicada ya en siete oportunidades- 1 o contradice ${ }^{4}$. Con ese respaldo, la cifra ya es aceptada y reforzada aun por analistas serios (García,2015), y se estiró aún más cuando se dijo que no correspondía a los abortos ilegales sino a los que ocurren en las tres circunstancias contempladas por la jurisprudencia para que la interrupción voluntaria del embarazo (IVE) sea legal (Ruiz, 2015).

Al parecer, los 400 mil abortos anuales surgieron en el país por regla de tres: el equivalente a la participación de la población en el total mundial, ajustado progresivamente al alza. Un indicio de ello es que antes de la estimación mundial nadie lanzaba números en los medios y después de difundir esa millonada, la proporción correspondiente al país fue adoptada por periodistas y activistas, hasta convertirse en coletilla obligada de cualquier referencia al tema.

E1 trabajo que presentó la primera estimación mundial de la OMS no se consigue, pero la sexta reedición tiene una sección metodológica reveladora que hace pensar en el cuento de García Márquez (WHO, 2008, 39). Es claro que los insumos para calcular los abortos clandestinos en el mundo son los comentarios y análisis disponibles en internet, no siempre elaborados con trabajo de campo ni con encuestas, sino mediante estimaciones que, como las de los periodistas colombianos, se derivan de cálculos de funcionarios con imaginación global que ven confirmadas sus profecías.

\section{INCOHERENCIAS CON LA INFORMACIÓN DISPONIBLE}

Cuando se empezó a abrir camino la cifra cercana al medio millón de abortos clandestinos anuales, ya existía en el país un estudio riguroso sobre aborto inducido, basado en una encuesta con una muestra respetable. Esta investigación, de la Universidad Externado de Colombia, no aventuró cifras anuales, pero mediante cálculos simples queda claro que la cifra era muy inferior: unos $130 \mathrm{mil}^{5}$, la tercera parte de la que pregonan medios y expertos. La proporción de 14,5

\footnotetext{
${ }^{4}$ La última sección examina el cálculo del número de abortos a partir de las ENDS.

5 En la muestra de más de 30 mil mujeres se reportaron menos de 10 mil abortos y unos 67 mil nacimientos, 14,5 abortos por cada cien hijos nacidos vivos. Suponiendo que esa fracción acumulada sirve para estimar el número de abortos ocurridos en un año con respecto a los nacimientos registrados en esa época, 900 mil, una simple regla de tres da esa cifra.
} 
abortos por cada 100 nacimientos reportados en esa muestra difiere notablemente de los más de 40 que supone el GI al calcular 400 mil.

Para una fracción más pertinente -embarazos que terminan en aborto $^{6}$ - ese estudio encontró que "el 12,37\% de esos embarazos terminó en aborto inducido" (ibíd., 40). De modo que en su apresurada estimación, Mónica Roa no solo mezcló el aborto espontáneo con el inducido sino que tampoco verificó que la proporción calculada por ella (27\%) difería de la del Externado. No obstante, sin contrastar fuentes, los medios atribuyeron la responsabilidad de esa cifra fantasiosa al Externado (ET, 2000b).

\section{Gráfica 1}

Interrupción voluntaria del embarazo en algunos países

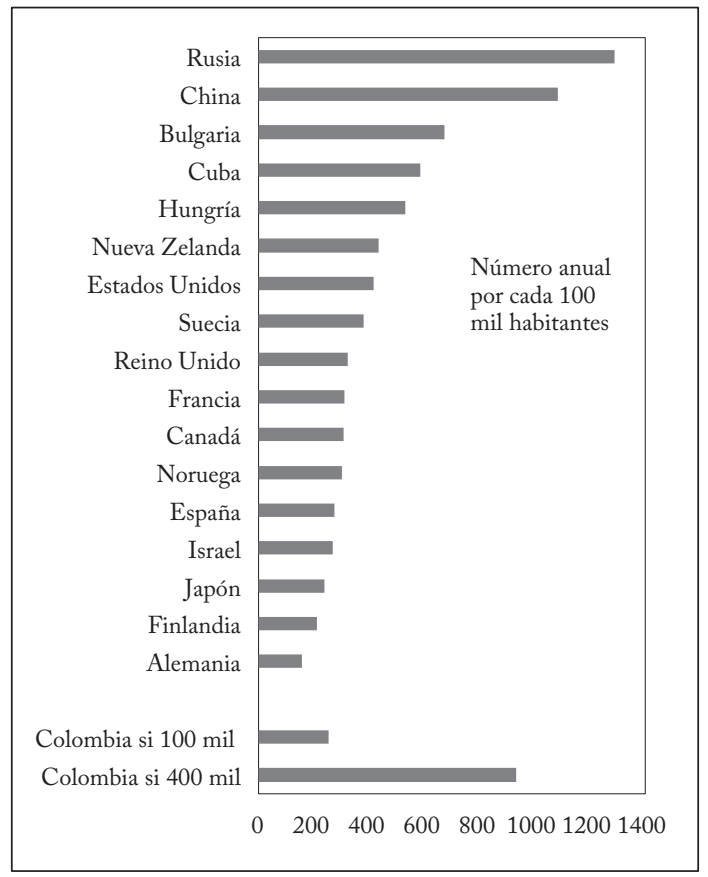

Fuente: www.infoplease.com/ipa/A0004397.html; para Francia, España y China: wikipedia.

Aun sin consultar ese trabajo, era evidente la discrepancia entre las cifras pregonadas y la información disponible, por ejemplo, con el número de abortos per cápita en países con alta incidencia de abortos. Durante varias décadas la Unión Soviética, donde se aprobó el aborto

6 "Una medida de la intensidad del fenómeno es la tasa de abortos por cada cien embarazos [...] mucho más fina porque no contiene los sesgos que introduce la mayor o menor exposición al riesgo de embarazo" (Zamudio et al., 1999, 40). 
libre en 1920, ocupó el primer lugar en interrupción de embarazos. En 1995, un despacho de una agencia española de noticias que se publicó en los medios colombianos mencionó su "récord en abortos" (Efe, 1995). En ese entonces, la población rusa era unas ocho veces mayor que la colombiana ${ }^{7}$, de modo que en términos per cápita el nivel de abortos en Colombia era supuestamente casi igual al de Rusia.

Otro país con alta incidencia de abortos es China, en buena parte debido a su estricta política demográfica y al aborto legalmente aceptado. "Uno de cada tres embarazos termina en aborto", escribió en 1993 el mismo periodista que no verificó que esa alta proporción era inferior a la que pregonaba párrafos antes (Santos, 1993). La simple comparación de las tasas de aborto per cápita de varios países habría puesto en duda la cifra de 400 mil abortos que carecía de sustento.

Las estimaciones para algunas ciudades colombianas también desafiaban el mito aceptado. "En Barranquilla se reportaron 1.267 abortos en 1992" 8 . Tomando ese dato como indicador, la cifra nacional solo sería de unos $43 \mathrm{mil}^{9}$. A finales de los noventa, los medios mostraron preocupación por el alto número de legrados en el Hospital Universitario del Valle (HUV): unos 4.000 al año (ET, 1998c). El HUV era el único hospital de Cali autorizado para practicar legrados, y se puede suponer que no solo llegaban casos de la capital sino de otros municipios del departamento. Ese número "alarmante" equivaldría a un total nacional de 40 mil a 80 mil abortos ${ }^{10}$. En 2000, un día antes de la publicación de un informe sobre embarazo adolescente se reportó el caso de un colegio distrital de Bogotá en el que "más de 40 niñas entre 13 y 17 años están embarazadas", y ninguna pensaba abortar $(E T, 2000 \mathrm{c})$. Este relato contradecía la idea de que la tercera parte de los embarazos, sobre todo de jóvenes, termina en aborto, la cual habría implicado unas doce interrupciones. Pero el redactor retomó sin dudar la cifra de 400 mil abortos.

\section{LOS ANTROS INSALUBRES DONDE MUEREN POCAS MUJERES}

Cualquiera de las incoherencias anteriores es menor frente a la más notoria, la astronómica cif ra de abortos clandestinos en condiciones de sanidad deplorables frente al bajo número de muertes provocadas por esa operación.

7290 millones de habitantes contra 37.

8 Según el Departamento Administrativo de Salud (Santos, 1993).

${ }^{9}$ En ese año, la población de esa ciudad era algo menos del 3\% de la del país [https://en.wikipedia.org/wiki/Barranquilla].

${ }^{10}$ En 1995 la población del Valle era el 10\% de la nacional y la de Cali el 5\% (Escobar y Collazos, 2007). 
La exagerada cifra de abortos mundiales se divulgó con otra estadística global: cientos de miles de mujeres muertas por interrupción clandestina del embarazo. "Por lo menos 200.000 mujeres mueren cada año por abortos fallidos", declaró un obstetra nigeriano en una publicación de la OMS a comienzos de los noventa (Ladipo, 1990). Oficialmente, la OMS era más cautelosa. $\mathrm{El}$ informe de un grupo técnico reunido en Ginebra en 1992 reconoció que era difícil determinar el número de muertes por aborto inseguro no solo por la imposibilidad de conocer la incidencia de aborto en mujeres en edad reproductiva sino porque no se sabía con precisión la proporción de casos que terminan fatalmente (WHO, 1992, 6). Ese mismo informe calculó un rango de 50 mil a 150 mil muertes y aclaró que "la eventual caída del número de muertes anuales relacionadas con abortos se puede atribuir a prácticas de cuidado más seguras" (ibíd., 7).

Cuando las estimaciones de mortalidad mundial por aborto se empezaron a divulgar en Colombia la cifra ya se había ajustado drásticamente a la baja: "70 mil mujeres mueren debido a complicaciones" (ET, 1996b); cifra respaldada por una de las revistas médicas más prestigiosas, The Lancet, que estimaba entre 60 mil y 120 mil muertes por aborto clandestino ${ }^{11}$.

La información sobre mortalidad materna se enmarcaba en dos teorías. La primera, difícil de rebatir, es que el aborto ilegal tiene más riesgos para la salud de la mujer. Incluso para esta hipótesis razonable hay escenarios que la contradicen. Uno es India, donde el aborto es legal pero se carece de una red sanitaria eficaz y se aborta "en un medio insalubre sin las medidas preventivas necesarias" (Efe, 1996a). Otro es Chile, donde en 1989 se derogó la ley que permitía el aborto terapéutico y, después de la ilegalización, entre 1989 y 2007 , "la mortalidad se redujo de 13,7 a 1,7 por 100.000 nacidos vivos" (Koch et al., 2012). Los datos colombianos sobre mortalidad materna provocada por aborto también muestran una caída sostenida, de 264 muertes en 1983 a 70 en 2010, un fuerte descenso bajo un régimen legal prohibitivo (DNP, 1999; Prada et al. 2011).

Estas experiencias sugieren que la clandestinidad no es una condición suficiente ni necesaria de alto riesgo de mortalidad por aborto, el cual depende menos de la ilegalidad que del cubrimiento

\footnotetext{
${ }^{11}$ Efe (1996a). Aunque no he identificado el artículo de The Lancet al que hace referencia esta agencia, cabe sospechar que fue obra de investigadores del GI y de la OMS que después publicaron sobre ese mismo tema en esa revista (Sedgh et al., 2008). La credibilidad del GI era y sigue siendo muy alta en Estados Unidos, un país donde las estadísticas se basan en fuentes confiables y verificables. En otros países las críticas a su metodología y a sus contradictorios supuestos son recientes (Crouse, 2008).
} 
y la calidad del sistema sanitario. Así empieza a reconocerlo la OMS. En la interrupción legal del embarazo -desde un aborto farmacológico en el hospital de un país desarrollado hasta la introducción de objetos en un consultorio no desinfectado de un país atrasado- hay "un espectro de riesgos, como la auto administración de misoprostol o el uso de técnicas anticuadas como el legrado, aun por médicos bien entrenados" (Ganatra et al., 2008). Con esa dispersión, sumada a la baja continua de la mortalidad por aborto, la OMS admite que sus definiciones "pragmáticas" - p. ej., considerar inseguro cualquier aborto no legal- se deben ajustar al nuevo contexto (ibíd.).

La segunda teoría - contraintuitiva, poco creíble y contraria a la evidencia y a los testimonios- es que el número de abortos es mucho mayor en países donde está penalizado ${ }^{12}$. Esa teoría insólita se propuso al comienzo con suma cautela ${ }^{13}$, pero se convirtió en verdad a fuerza de repetirla. Nunca se intentó comprobarla, considerando por ejemplo el mayor acceso a contraceptivos en países desarrollados. Y quedó flotando una asociación bizarra: la clandestinidad como incentivo para que las mujeres aborten y para que los médicos of rezcan ese servicio.

La experiencia de España, donde el aborto se legalizó informalmente en 1985, contradice la pretensión de que la ilegalidad se asocia a una mayor incidencia del aborto. En este caso, casi un experimento controlado, se observa lo contrario: a medida que el aborto se percibía legalmente autorizado su demanda aumentaba y generaba la oferta para satisfacerla ${ }^{14}$.

En Colombia, ya en la primera mitad de los noventa la tasa de mortalidad materna por todas las causas (DNP, 1999) $)^{15}$, con una marcada tendencia a la baja, era inferior a 10 por cada $100 \mathrm{ml}$ nacimientos (ibíd., cuadro 6, 20). Así, las cifras calculadas por las agencias inter-

${ }^{12}$ Para América del Sur y África Oriental, donde el aborto está penalizado, el GI estima 33 y 39 abortos por cada mil mujeres, mientras que en Europa Occidental, donde es legal, la cifra observada es de apenas 12 (Singh et. al, 2009, gráfica 3.3, 19).

13 "Los expertos calculan que esta cifra, muy superior a la de los países desarrollados, está directamente relacionada con la legalidad del procedimiento, aunque indicaron que esto no es una garantía" (Reuter, 1996).

${ }^{14}$ En 1987, dos años después de aprobada la ley que lo despenalizó en tres circunstancias excepcionales, se registraron menos de 17 mil abortos. En los años siguientes hubo leves aumentos anuales que se estabilizaron en los noventa. Desde 2000 el aumento fue sostenido y llegó a un máximo de 118 mil en 2011, un año después de sustituir el sistema de prohibición con restricciones por uno de plazos (Koch et al., 2012), Las causas o excepciones existentes entre 1985 y 2010 eran burladas sistemáticamente por las clínicas especializadas que certificaban que la salud mental de la mujer sería afectada si no abortaba (Rubio, 2015).

${ }^{15}$ Fuera de abortos, toxemias, hemorragias, complicaciones de parto, perperio y otras. 
nacionales eran un 60\% mayores que las causadas por esa y las demás razones. El número de mujeres muertas por aborto era apenas superior a $100^{16} \mathrm{y}$, con altibajos, la participación del aborto en la mortalidad materna venía disminuyendo. El mito de 400 mil abortos ignora esa tendencia a una mortalidad cada vez menor.

Gráfica 2

Mortalidad materna, 1983-1995

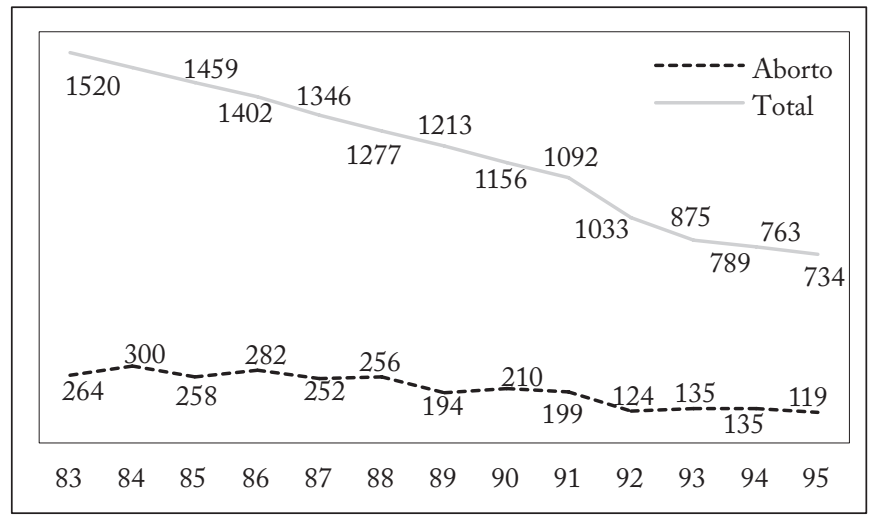

Participación del aborto en la mortalidad

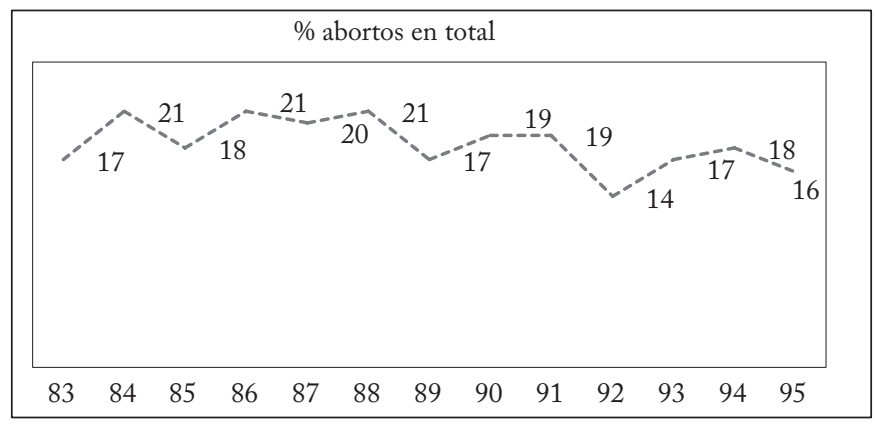

La información sobre el continuo y notable descenso de las muertes por aborto no se limitaba a documentos técnicos a los que pocos tenían acceso. En 2001 el principal diario nacional anunció: "las muertes maternas bajaron el 51 por ciento en Bogotá, en los últimos 10 años" (ET, 2001a).

Los medios divulgaron datos de algunos hospitales que atendían mujeres con complicaciones por aborto clandestino, que se pueden usar para calcular la relación entre muertes e interrupciones de embarazo y, con ella, el total de abortos en el país. En 1998, el jefe de la unidad de gineco-obstetricia del HUV calculó que morían 12 de ${ }^{16}$ En el quinquenio 1990-1995, el promedio fue de 154; 119 en 1995. 
cada 4.000 mujeres que allí se atendían por complicaciones de aborto $(E T, 1998 \mathrm{c})$. Con esa proporción, el total nacional de interrupciones de embarazo sería de $50 \mathrm{mil}$, un número no muy diferente del que indican otros datos disponibles.

\section{UNA TRAGEDIA PEOR QUE EL CONFLICTO ARMADO}

Cuando se empezó a imponer la cifra de varios cientos de miles de abortos en el país, también llegó del extranjero una tasa de mortalidad por aborto inconsistente con el número mundial de muertes maternas que se pregonaba ${ }^{17}$. Si esa proporción se hubiese aplicado a la cifra mundial consagrada por los medios -20 millones-, las muertes anuales por esa causa habrían llegado a 3,2 millones. Las agencias internacionales nunca superaron la barrera de 200 mil y habían estabilizado la cifra de defunciones maternas por esa causa en 70 mil. Al multiplicar la tasa pregonada internacionalmente por el supuesto número de abortos colombianos se llegaba a una cifra de muertes espeluznante: "con base en los 300 mil casos que revela el Ministerio de Salud, y considerando la estadística de la Unicef, alrededor de 45 mil mujeres al año podrían morir en el país debido a abortos mal practicados" (ibíd.).

En esos días, el número de homicidios no llegaba a 28 mil, pero eso no despertó el escepticismo y el sentido crítico del periodista. En cambio, añadió que la cifra calculada de mujeres que podrían estar muriendo por abortos clandestinos "duplica el número de víctimas que arroja anualmente la violencia en sus múltiples manifestaciones: guerrilla, narcotráfico y delincuencia común" (ibíd.). Para un total mundial de 70 mil muertes por aborto, Colombia aportaba más de la mitad. Ese despropósito tampoco hizo sospechar al periodista.

\section{LA CONTABILIDAD DE ACTIVIDADES CLANDESTINAS}

Desde sus inicios, uno de los principales problemas de la criminología fue medir la magnitud del fenómeno en estudio (Rubio, 2008). Hacia 1930, algunos sociólogos estadounidenses mostraron que la información judicial o de policía era insuficiente para hacer diagnósticos, pues no daba información de la delincuencia no denunciada, la "cifra negra" de la criminalidad.

El registro de abortos, cuando son ilegales, tiene los mismos problemas que el registro de otros delitos, más acentuados. A diferencia

\footnotetext{
17 "Según la Unicef, 17 de cada cien mujeres que recurren a esta práctica clandestina mueren debido a las precarias condiciones en las que se lleva a cabo" (Santos, 1993).
} 
de un crimen que la víctima puede tener interés en reportar, nadie denuncia un aborto, salvo en casos excepcionales como la muerte de la mujer sometida a la operación. Como ya se señaló, hay numerosos testimonios de personas involucradas en un aborto que lo callan; por eso no se registra. Así, su incidencia se debe estimar a partir de las pocas trazas que quedan, con supuestos heroicos para cuantificar la fracción que no deja rastros.

Uno de los métodos para hacer estas estimaciones se basa en los abortos clandestinos que, por complicaciones, llegan al sistema de salud. El GI lo usa desde hace varias décadas. El supuesto básico es que el personal del sistema de salud que atiende esas complicaciones tiene una idea aproximada de los abortos que no necesitaron atención posterior y no se registraron. Así, al número de complicaciones se aplica un multiplicador basado en la opinión del personal de salud que habría podido atenderlos. Ese factor normalmente se asocia, en forma inversa, a los riesgos de abortar, los que a su vez dependen de la tecnología predominante para interrumpir el embarazo. Si el aborto ilegal se vuelve más seguro cabe esperar menos complicaciones atendidas por el sistema sanitario y un multiplicador mayor. Esto en teoría, pues si los médicos no distinguen un aborto inducido de uno espontáneo, y la atención médica cubre a toda la población, las mujeres pedirán atención aun después de un aborto farmacológico sin grandes complicaciones. Y cabe pensar que con el uso del misoprostol esa es la situación en Colombia.

\section{Diagrama 1}

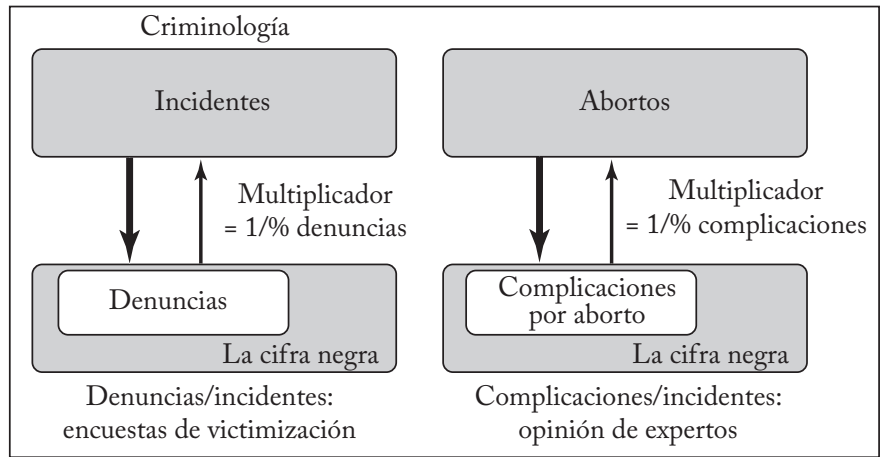

No se han discutido suficientemente dos aspectos de ese método. El primero, los mecanismos a través de los cuales quienes atienden las complicaciones reciben información de los abortos clandestinos que no requirieron atención posterior. Ya se mencionó la férrea voluntad 
de no dejar ningún rastro en caso de aborto, y si no requiere atención médica posterior, es difícil imaginar cómo llega esa información al sistema de salud, cuyo personal nada puede saber de quienes no piden atención hospitalaria. En el trabajo de campo realizado por el Externado se observó que había vasos comunicantes entre médicos, enfermeras o farmaceutas que practicaban o facilitaban el aborto y el sistema de salud, pero que iban en una sola dirección: por ejemplo, una recomendación para que, de haber complicaciones -hemorragias, infección, etc.- la mujer fuera a una clínica u hospital para que la atendieran. Pero no en sentido contrario. Tampoco hay testimonios de la mecánica de blanqueo de esa información confidencial.

El segundo aspecto, esencial en el escepticismo de la criminología hacia las cifras oficiales de delitos, es el posible interés de quienes las manejan por aumentarlas o reducirlas a conveniencia. Ese parece ser el caso del trabajo realizado por el GI para su publicación de 2011, cuyo número estimado de abortos es sospechosamente similar al que ya habían adoptado los activistas y los medios. Luego de señalar que en un amplio trabajo de campo se contabilizaron 93 mil complicaciones por aborto en el sistema sanitario, aplica un extraño pero conveniente multiplicador de 4,29 y llega a una cifra: 398.700 abortos. Además de opaco, el multiplicador del GI es elástico ${ }^{18}$. El escepticismo lo corrobora un artículo anterior de ese instituto que justificó el ajuste hacia arriba debido a que los riesgos del aborto clandestino se habían reducido notablemente entre 1976 y 1992, y llegaban menos casos al sistema de salud ${ }^{19}$. Desde 1992, con el avance del aborto farmacológico, los riesgos siguieron bajando, quizá rápidamente; y el multiplicador ha debido aumentar. Extrapolando la tendencia anterior, hoy su valor estaría entre 8 y 9 , y los abortos así estimados con ese método serían unos 800 mil, una cifra impresentable, casi igual a la de nacimientos. Un multiplicador exacto de 4,29 daba una cifra igual a la pregonada, aunque un valor tan bajo suponía implícitamente que el riesgo de abortar aumentó desde 1986, algo que contradice ese mismo trabajo y toda la evidencia disponible. Según ese estudio, en todo el país, incluida Bogotá, hoy es más inseguro abortar que en 1986. La homogénea distribución geográfica del multiplicador también incomoda. A zonas con incidencia de abortos y acceso a los servicios de salud muy diferentes se les aplica un factor de expansión similar.

\footnotetext{
${ }^{18}$ Un año antes, el GI empleó un multiplicador de 4,12 (Singh, Prada y Juárez, 2010)

${ }_{19}$ Allí se usó un multiplicador de 5,5, que reajustó el valor de 3,5 años atrás debido a "adelantos en la disponibilidad de servicios seguros" (Singh y Sedgh, 1997).
} 
Aunque otros artículos del GI reconocen que el uso del misoprostol afecta la estimación del total de abortos a partir del número de mujeres que acuden al sistema de salud ${ }^{20}$, la ratificación de la cifra mítica recibió amplio cubrimiento en la prensa; mientras que una cifra de ese trabajo, 70 muertes anuales por aborto -que confirma el descenso del riesgo-, recibió menos atención.

Los problemas de la estimación mediante este multiplicador no se limitan a ese discutible y oportuno valor. También se ha criticado la cifra de 93 mil complicaciones ${ }^{21}$. Una imprecisión más grave es que de las 115 mil mujeres atendidas por cualquier tipo de aborto, se calculó que 22 mil correspondían a pérdida espontánea usando la proporción de mujeres con aborto espontáneo entre las que dan a luz en una IPS. Esto supone que por cada mujer hospitalizada por pérdida espontánea más de cuatro van por aborto inducido, una relación que contradice la evidencia de varios países, incluida la de Colombia ${ }^{22}$.

Las cifras del GI sobre el aborto en Colombia no son las únicas que suscitan graves interrogantes. En otros países se ha constatado su exageración. Para 2006, calculó 879 mil abortos anuales en México; 165 mil en la capital (GI, 2008). El aborto se legalizó en México D.F. en 2007. Y entre abril de ese año y septiembre de 2011, allí se practicaron $67 \mathrm{mil}$ interrupciones legales de embarazo (ILE), algo más de 15 mil al año, menos de la décima parte de la cifra del GI. Esa sobrestimación, esta vez verificada, es similar a la de Colombia.

E1 GI no parece haber estimado por aparte el número de abortos en Uruguay. Pero con la tasa que usa para América Latina -33 abortos por cada 1.000 mujeres entre 15 y 44 años- habría unos $23 \mathrm{mil}^{23}$. A finales de 2012 se despenalizó el aborto antes de las 12 semanas de gestación. En 2013, el primer año de vigencia de la ley, se registraron 6.676 interrupciones de embarazo (La Nación, 2015), casi la tercera parte de la cifra estimada con los parámetros del GI.

${ }^{20} \mathrm{Y}$ recomiendan tres métodos alternativos, ninguno de los cuales se aplicó en la apresurada ratificación de 400 mil abortos en 2011, aunque el informe reconocía que el misoprostol se usaba en el 50\% (ver, p. ej., Wilson et al., 2010).

${ }^{21}$ Esa cifra se estimó con base en una encuesta de opinión a 289 personas. E1 primer problema se refiere a la representatividad de esa muestra. "Los autores no mencionan la probabilidad de servicios postaborto [...] ni las razones que justifican las proporciones de la primera elección [...] la sucesiva exclusión finaliza en 289 instituciones seleccionadas. La representatividad de la muestra es por tanto desconocida y parece mejor ajustada a un muestreo por conveniencia". E1 segundo es que el número de complicaciones no se basó en registros (Koch et al., 2012, 363).

${ }^{22}$ Más adelante se presentan estimaciones de esta relación.

${ }^{23}$ Datos de población por sexo y edad tomados de INE, [http://www.ine.gub. uy/web/guest/censos-2011]. 


\section{EL ABORTO EN LAS ENCUESTAS DE DEMOGRAFÍA Y SALUD}

Una opción para estimar la magnitud de un delito, usual en criminología, son las encuestas de población que preguntan si se ha sido víctima de un acto criminal y suelen preguntar si se puso en conocimiento de las autoridades. Aunque menos extendidas, también son corrientes las encuestas confidenciales y anónimas, de "auto reporte", a grupos de infractores -en general jóvenes- que indagan el tipo y la cantidad de delitos que han cometido, y comparan sus respuestas con las de un grupo de control.

Para el aborto, el instrumento de medición pertinente se asemeja a las encuestas de auto reporte, como la encuesta anónima del Externado ${ }^{24}$, cuya principal pregunta era " $¿ S e$ ha practicado o se ha mandado practicar alguna vez un aborto?”. Esa redacción elimina la posible confusión entre aborto inducido y espontáneo.

Otra fuente de información directa, de mujeres que han abortado, son las ENDS, que incluyen una pregunta sobre aborto en todas sus versiones desde 1990. Entre ellas y la del Externado hay tres diferencias que impiden comparar sus resultados. La principal es que en las ENDS la pregunta se responde al encuestador y no en secreto. Es razonable pensar que un acto que no se quiere comentar al grupo más íntimo tampoco se quiera informar a un desconocido, menos aún si se quiere superar y olvidar rápidamente. La segunda diferencia es que el cuestionario de las ENDS es mucho más extenso y cubre más aspectos. E1 hecho de que no se limiten al aborto puede aumentar el subregistro, pues distrae y hace posible evadir esa pregunta incómoda. La tercera, quizá más fácil de resolver, es que las primeras ENDS, de 1990 a 2000, mezclaban abortos inducidos y pérdidas espontáneas: “'Tuvo alguna vez un embarazo en el cual tuvo pérdida, abortó o terminó en un nacido muerto?”, y solo se podía responder sí o no. Desde 2005, a la pregunta se puede responder si hubo pérdida, interrupción, embarazo extrauterino o muerte fetal intrauterina.

Debido a esas razones no es prudente comparar los resultados de la encuesta del Externado con los de las ENDS, cuya metodología es más homogénea y permite hacer comparaciones entre periodos. El único comentario sólido es que en la encuesta realizada por el Externado habría un subregistro menor del aborto inducido.

${ }^{24}$ Cuyo cuestionario aclara que es "de autodiligenciamiento secreto y consignación reservada”. La mujer lo respondía y lo depositaba en un sobre sin que el encuestador pudiera ver las respuestas. Por ello tiene pocas preguntas y cubre pocos aspectos ajenos a la fecundidad y la interrupción de embarazo (Zamudio et al., 1999, Anexo). 
Para conocer la proporción de delitos que llegan a conocimiento de las autoridades, las encuestas de victimización preguntan a las víctimas si los denunciaron. Para comparar la información de las encuestas sobre aborto y los registros del sistema de salud de mujeres que pidieron atención médica por complicaciones, hay que preguntar si después del aborto acudieron al servicio de salud. Ni las ENDS ni la encuesta del Externado hacen esa pregunta, que permite evaluar qué tan adecuado es el multiplicador que usa el GI. La información más directa que se obtiene de las ENDS es el porcentaje de mujeres que alguna vez han tenido una interrupción de embarazo (IE), incluidas pérdidas espontáneas y abortos inducidos (gráfica 3).

Gráfica 3

Proporción de mujeres que han tenido interrupción de embarazo

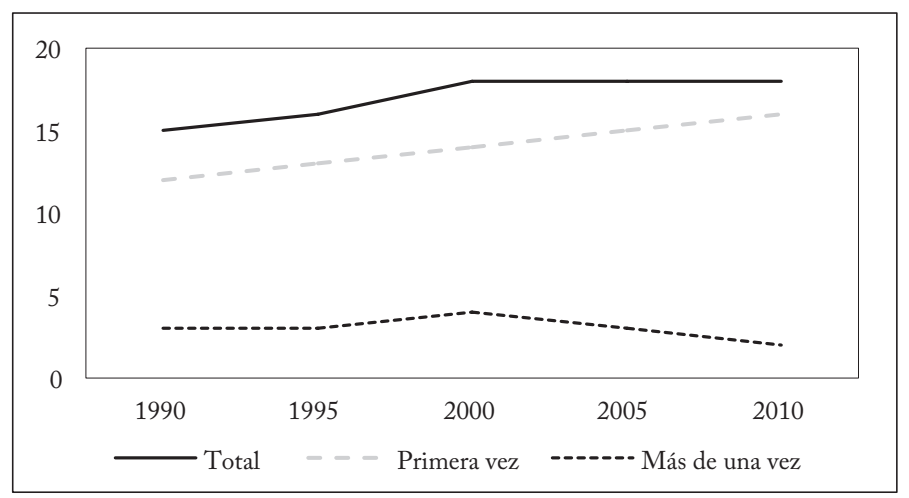

Incluye pérdidas y abortos.

Fuente: ENDS.

La cifra total muestra un aumento visible -del 15\% en 1990 al 18\% en 2000- y se estabiliza desde 2010. Cuando se descompone entre mujeres que reportan una interrupción de embarazo y más de una, la estabilización de la segunda mitad del periodo es resultado de un crecimiento similar al de la primera mitad para quienes reportan una sola interrupción y un descenso del $4 \%$ al $2 \%$ del porcentaje que reporta más de una. Para usar estos porcentajes en la estimación del número de abortos en el país es necesario hacer algunos supuestos, que se resumen en el cuadro 1.

En cada encuesta se tiene el número de mujeres de la muestra y el de las que han interrumpido el embarazo. Se sabe cuántas han tenido una IE única (IEU) y cuántas más de una (IEMU). Del trabajo del Externado se puede tomar el número de abortos de las mujeres que reportan más de uno para calcular la proporción de IEU e IEMU 
con respecto al total de nacimientos reportados en cada encuesta ${ }^{25}$. Si esa proporción se aplica a los nacimientos ocurridos en cada año se obtiene el total de IE en el país, desagregado entre únicas y múltiples.

Cuadro 1

Estimación del número de abortos

\begin{tabular}{lrrrrr}
\hline & 1990 & 1995 & 2000 & 2005 & 2010 \\
\hline Mujeres en la muestra & 8.644 & 11.136 & 11.585 & 41.344 & 53.521 \\
Con IE & 1.305 & 1.734 & 2.141 & 7.241 & 9.778 \\
Porcentaje con IE & 15,1 & 15,6 & 18,5 & 17,5 & 18,3 \\
Porcentaje de ellas con más de una IE & 17,6 & 17,5 & 19,7 & 14,8 & 13,4 \\
Mujeres con IEU & 1.076 & 1.431 & 1.719 & 6.172 & 8.472 \\
Mujeres con IEMU & 229 & 303 & 422 & 1.069 & 1.306 \\
IE por mujer con IEMU & 2,3 & 2,3 & 2,3 & 2,3 & 2,3 \\
Número de IEU & 1.076 & 1.431 & 1.719 & 6.172 & 8.472 \\
Número de IEMU & 527 & 698 & 970 & 2.460 & 3.005 \\
Nacimientos reportados en la muestra & 15.976 & 21.830 & 21.237 & 71.278 & 91.399 \\
IEU/nacimientos & 6,7 & 6,6 & 8,1 & 8,7 & 9,3 \\
IEMU/nacimientos & 3,3 & 3,2 & 4,6 & 3,8 & 3,3 \\
Nacimientos en el país (miles) & 955 & 937 & 926 & 922 & 910 \\
Número de IEU(miles) & 64 & 61 & 75 & 80 & 84 \\
Número de IEMU(miles) & 32 & 30 & 42 & 32 & 30 \\
Total de IE (miles) & 96 & 91 & 117 & 112 & 114 \\
\hline
\end{tabular}

Fuente: ENDS, 1990-2010.

Gráfica 4

Interrupciones de embarazo cada año

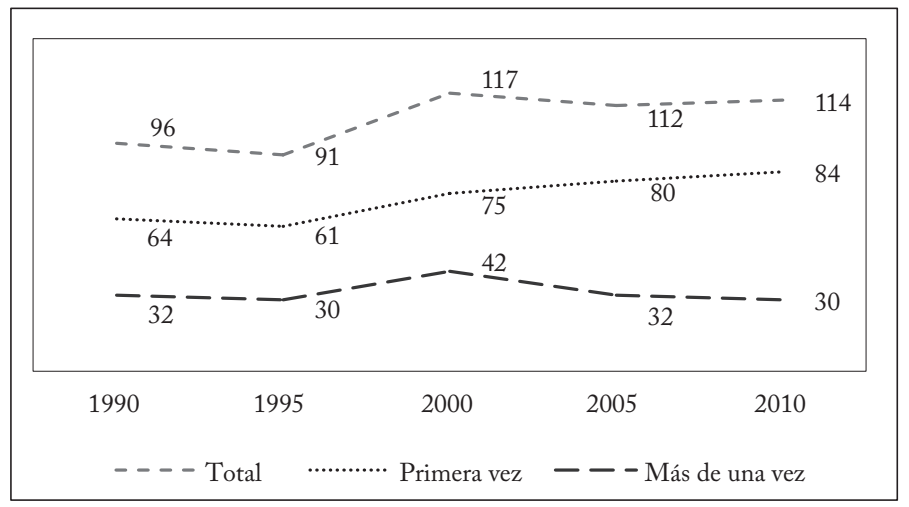

Así, las IE -espontáneas e inducidas-derivadas de las ENDS son un poco más de 100 mil. Si se tienen en cuenta los datos de las encuestas de 2005 y 2010 -el $55 \%$ por pérdidas y solo el $30 \%$ por aborto ${ }^{26}$ - el número total de interrupciones voluntarias estaría entre 30 y $40 \mathrm{mil}$ anuales; una cifra diez veces menor que los 400 mil tan pregonados. Cabe destacar que el orden de magnitud de esta estimación es muy

${ }^{25}$ Se supone que el número de IE de las que han tenido más de una es similar al de las repitentes.

${ }^{26}$ Ver la parte inferior derecha de la gráfica 4. 
similar al que se obtiene con fuentes muy diferentes, como los abortos estimados en el HUV de Cali y en Barranquilla. Y muy cercano al que se calcula usando las únicas cifras oficiales relativamente confiables, las de mortalidad materna.
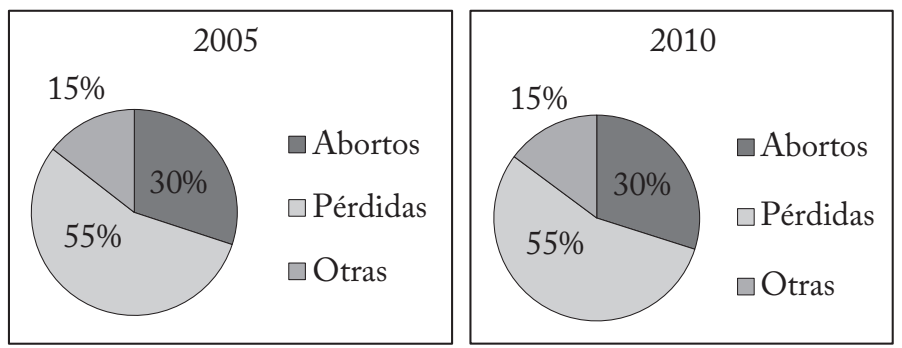

Incluye pérdidas y abortos.

Fuente: ENDS.

$\mathrm{E} 1$ orden de magnitud del total de interrupciones reportadas en las ENDS de 2005 y 2010 es similar al de las complicaciones calculadas por el GI mediante la encuesta al personal de salud (93 mil) con las que ratificó la cifra de 400 mil abortos. Teniendo en cuenta el testimonio común de los médicos de que al atender a una mujer que pide atención postaborto es casi imposible diferenciar entre interrupción espontánea o inducida - una dificultad que han agravado las técnicas farmacológicas-, se puede pensar que el sentido original del multiplicador y su utilidad para estimar el número total de abortos han cambiado radicalmente. Ya no es tan extraña la situación -que ese método no contempla- de un aborto practicado fuera del sistema de salud (cada vez más en el domicilio de la mujer) que, a pesar de su bajo riesgo, lleva a solicitar atención médica posterior.

E1 estudio del Externado encontró que de cada 100 embarazos, 82 resultaba en nacimientos, 12 en aborto inducido y 6 aborto en espontáneo (Zamudio et al., 1999, cuadro 18, 41). O sea que, a comienzos de los noventa, por cada pérdida había dos interrupciones voluntarias. Diez años después, en la ENDS de 2005, la proporción prácticamente se había invertido, pues por 30 abortos voluntarios se reportaron 55 espontáneos. Así, parece ser que la confusión entre interrupción inducida y espontánea es cada vez más común, aun entre las mujeres que responden una encuesta.

Los menos de 50 mil abortos anuales que resultan de las ENDS sin duda subestiman la cifra real porque el formulario no se diligencia de manera anónima, lo que implica mayor reticencia a reportar un acto punible. También es posible que la extensión del formulario y el 
hecho de que aborde otros temas acentúen la inclinación a no mencionar algún aborto. La manifestación más obvia de este subregistro es la mayor proporción de mujeres que en la encuesta corta y secreta del Externado aceptó en 1992 haber abortado alguna vez (22,9\%) frente a la que en las ENDS de años cercanos reportó haber tenido interrupciones de embarazo (15\% en 1990 y 19\% en 1995).

\section{Gráfica 5}

Interrupciones de embarazo cada año

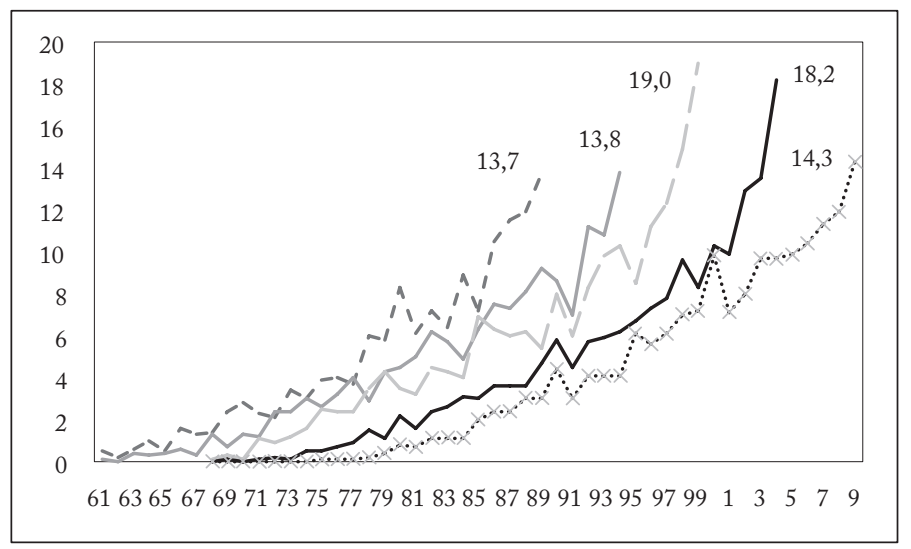

Total por cada 1.000 mujeres, dato reportado en la encuesta.

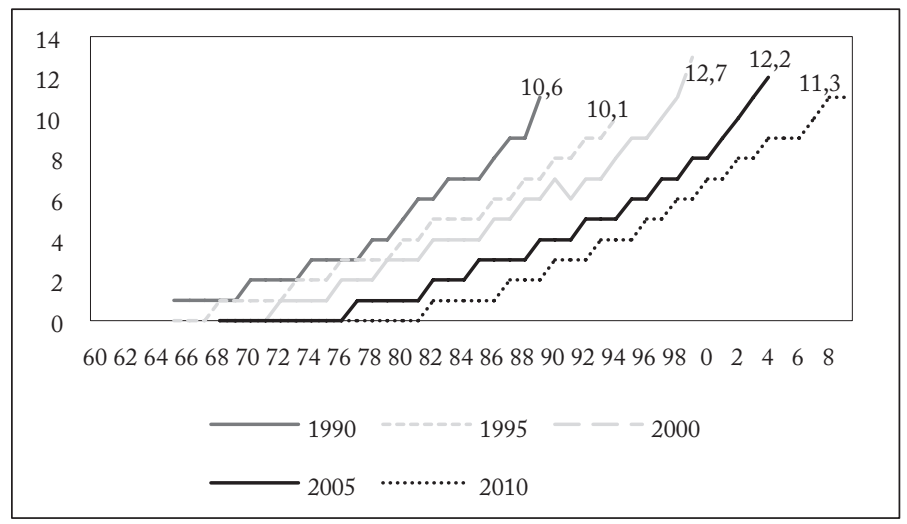

Total por cada 1.000 mujeres, promedio móvil quinquenio anterior.

Incluso sin esta comparación que evidencia el subregistro, en las mismas ENDS hay indicios de subregistro. A las mujeres que reconocen haber abortado se les pregunta en qué fecha. Si el reporte fuera fidedigno, esa pregunta daría indicios del número de abortos ocurridos en cada uno de los años anteriores. Pero el perfil temporal 
de las interrupciones de embarazo según recuerdan las mujeres que respondieron las distintas versiones de la encuesta es peculiar y tiende a concentrarse en el periodo más reciente, de modo que los abortos parecen dispararse justo antes de cada encuesta.

Esta información deja en claro dos cosas. Primera, la subestimación de abortos reportados aumenta con el tiempo: en todas las versiones de la encuesta el número de abortos reportados para años atrás es menor que para el año de la encuesta. Aunque parte de ese menor número puede obedecer a que las mujeres de mayor edad se excluyen de las encuestas, es evidente que el "olvido" de los abortos aumenta con el paso de los años. Segunda, los datos más recientes - p. ej., los del año o el quinquenio anterior a la encuesta- muestran entre sí un patrón similar al de los datos globales: dos periodos relativamente estables, 1990-1995 y 2000-2010, separados por un visible aumento entre 1995 y 2000.

Para corregir en parte el subregistro en cada versión se podría remplazar el promedio anual de abortos reportado por el número del año anterior a la encuesta -y obtener así una cifra máxima- o por el promedio de quinquenio anterior a la encuesta suponiendo que es el valor medio. E1 número de abortos reportado en cada encuesta se tomaría como el valor mínimo del rango probable.

\section{Gráfica 6}

Rango de interrupciones de embarazo cada año

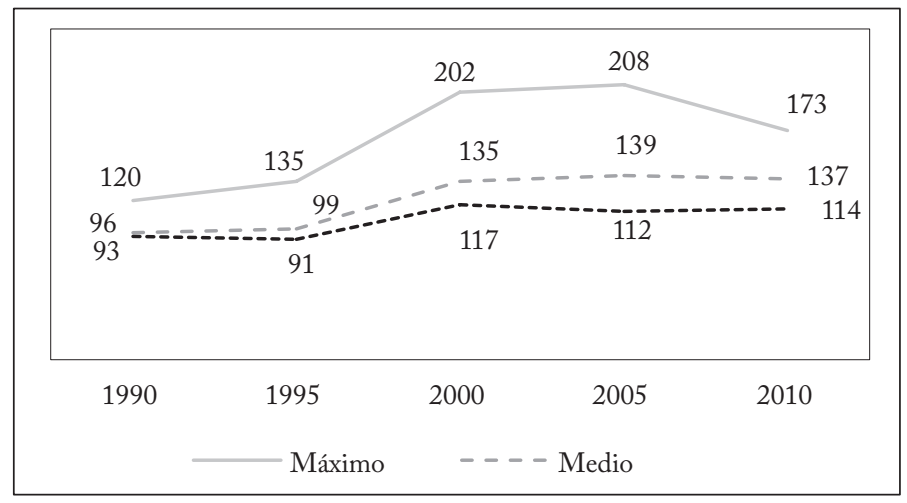

Incluye pérdidas y abortos.

Fuente: ENDS.

Este ejercicio muestra que el periodo de mayor incertidumbre en el reporte de abortos inducidos fue la primera mitad de la última década. Además, puesto que hoy se reportan como pérdidas espontáneas algunos abortos, se puede suponer que su participación respectiva es del 
$66 \%$ y el 33\% -un supuesto conservador pues son las cifras que obtuvo el Externado hace más de dos décadas-, y después de compensar el "olvido" o la reticencia, se obtendría un rango de $75 \mathrm{mil} \mathrm{a} 114 \mathrm{mil}$ abortos anuales, con un valor medio de 90 mil. Menos de la cuarta parte de la pertinaz cifra de 400 mil que ninguna evidencia corrobora.

\section{REFERENCIAS BIBLIOGRÁFICAS}

1. Celis, F. Colombia erótica, Bogotá, Intermedio Editores, 2002.

2. Crouse, J. S. "Planned parenthood think tank inflates abortion numbers", Washington Times, 27 de diciembre de 2008.

3. DNP. "Mortalidad materna", Bogotá, Unidad de Desarrollo Social, Boletin 22, 1999, [https://colaboracion.dnp.gov.co/CDT/Desarrollo\%20 Social/boletin22.pdf].

4. EE. "En Colombia hay un médico por cada 846 habitantes", El Espectador, 27 de febrero de 2012.

5. Efe. "Rusia récord en abortos", El Tiempo, 23 de junio de 1995.

6. Efe. "Vaticano. Cifras de abortos", El Tiempo, 23 de abril de 1996.

7. Efe. "El aborto cobra 100 mil víctimas al año", El Tiempo, 15 de junio de 1996a.

8. Escobar, J. y J. A. Collazos. "Series históricas del departamento del Valle del Cauca: un compendio de herramientas para la investigación regional", Ensayos sobre Economía Regional 47, 2007.

9. ET. "Adiós a discriminación de la mujer", El Tiempo, 29 de mayo de 1992.

10.ET. "Latinoamérica aborta por montones", El Tiempo, 28 de abril de $1994 \mathrm{a}$.

11.ET. "E1 aborto, en serio", El Tiempo, 22 de agosto de 1994b.

12. ET "Alarmantes cifras de aborto en Cali", El Tiempo, 19 de abril de $1996 a$.

13. ET. "Aborto, el lado oscuro de las madres", El Tiempo, 8 de mayo de 1996 b.

14. ET. "Alarmantes cifras de aborto en Cali", El Tiempo, 19 de abril de $1996 \mathrm{c}$.

15. ET. "Buscan prevenir el aborto en Bogotá", El Tiempo, 29 de septiembre de 1998a.

16. ET. "Renace debate para despenalizar el aborto", El Tiempo, 7 de diciembre de 1998b.

17. ET. "Práctica de abortos no se detiene en Cali", El Tiempo, 27 de marzo de 1998c.

18. ET. "Primera condena por aborto", El Tiempo, 6 de julio de 2000a.

19. ET. "Los médicos del aborto", El Tiempo, 29 de octubre de 2000b.

20. ET. "Aborto, un drama en las aulas escolares", El Tiempo, 29 de octubre de 2000c.

21. ET. "Ahora hay menos abortos", El Tiempo, 30 de mayo de 2001a.

22.ET. "Aumentó embarazo de adolescentes", El Tiempo, 25 de septiembre de 2001b. 
23. Fernández, S.; G. Gutiérrez T. y R. Viguri U. "La mortalidad materna y el aborto en México", Boletín médico del Hospital Infantil de México $69,1,2012$.

24. Ganatra. B.; Ö. Tunçalp et al. "From concept to measurement: Operationalizing WHO's definition of unsafe abortion”, Bulletin of World Health Organization 92, 3, 2014, p. 155.

25. García V., M. "La campaña de Profamilia”, El Espectador, 29 de mayo de 2015.

26. GI. "Datos sobre el aborto inducido en México", 2008, [http://www. guttmacher.org/pubs/2008/10/01/FIB_IA_Mexico_sp.pdf

27. Juárez, F.; S. Singh et al. "Estimaciones del aborto inducido en México: ¿qué ha cambiado entre 1990 y 2006?”, Perspectivas Internacionales en Salud Sexual y Reproductiva, 2009, [https://www.guttmacher.org/ pubs/journals/3500409S.pdf].

28. Juárez, F.; J. Cabigon y S. Singh. "The sealed envelope method of estimating induced abortion: How much of an improvement?", S. Singh, L. Remez y A. Tartaglione, Methodologies for estimating abortion incidence and abortion-related morbidity: A review, 2010, pp. 107-123.

29. Koch, E.; M. Bravo et al. "Sobrestimación del aborto inducido en Colombia y otros países latinoamericanos", Ginecología y Obstetricia de México 80, 5, 2012, pp. 360-372.

30. La Nación. "Uruguay: el número de abortos legales aumentó un 20 por ciento en 2014", 29 de marzo de 2015.

31. Ladipo, O. "Unsafe abortion in the Third World", World Health, abrilmayo de 1990, [http://apps.who.int/iris/bitstream/10665/52227/1/ WH_4-5_1990_p20_eng.pdf].

32. Lamas, $\bar{M}$. “La despenalización del aborto en México”, Nueva Sociedad 220, 2009, [http://www.plataformademocratica.org/Publicacoes/22841. $\mathrm{pdf}]$.

33. Mera, A. "Mortalidad materna vuelve a dispararse en Colombia", El Pais, 26 de abril de 2014.

34. Moanack, G. "E1 aborto: una realidad social”, Portafolio, 16 de agosto de 2000.

35. Prada, E. et al. Embarazo no deseado y aborto inducido en Colombia: causas y consecuencias, Nueva York, Guttmacher Institute, 2011, [http:// www.guttmacher.org/pubs/Embarazo-no-deseado-Colombia.html].

36. Prada, E.; A. Biddlecom y S. Singh. "Aborto inducido en Colombia: nuevas estimaciones y cambios de 1989 a 2008", Perspectivas Internacionales en Salud Sexual y Reproductiva, 2012, [http://www.guttmacher. org/pubs/journals/3800212S.pdf].

37. Remez, L. "E1 aborto clandestino: una realidad latinoamericana", Perspectivas Internacionales en Planificación Familiar, 1995, [http:// www.guttmacher.org/pubs/journals/21spa02995.pdf].

38. Reuter. "Cada minuto hay 85 abortos en el mundo", El Tiempo, 15 de febrero de 1996.

39. Roa, M. "E1 problema no es solo de estadísticas", Semana, 3 de diciembre de 2005.

40. Rubio, M. "Evaluación de las leyes: lecciones de la criminología", Revista de Economia Institucional 10, 19, 2008, pp. 131-160. 
41. Ruiz-N., C. “Cosas de niñas”, El Espectador, 27 de mayo de 2015.

42. Santos R., A. "Aborto: asunto de vida o muerte", El Tiempo, 17 de enero de 1993.

43. Sedgh, G.; S. Singh et al. "Induced abortion: Incidence and trends worldwide from 1995 to 2008", The Lancet, febrero de 2008.

44. Singh, S. y G. Sedgh. "Relación del aborto con las tendencias anticonceptivas y de fecundidad en Brasil, Colombia y México", Perspectivas Internacionales en Planificación Familiar, 1997, [http://www. guttmacher.org/pubs/journals/23spa00297.pdf].

45. Singh, S.; D. Wulf et al. "Abortion worldwide: A decade of uneven progress", 2009, [https://www.guttmacher.org/pubs/AbortionWorldwide.pdf].

46. Singh, S.; L. Remez y A. Tartaglione. Methodologies for estimating abortion incidence and abortion-related morbidity: A review, 2010, [http:// www.guttmacher.org/pubs/compilations/IUSSP/abortion-methodologies. pdf].

47. Singh, S.; E. Prada y F. Juárez. "The abortion incidence complications method: A quantitative technique", S. Singh, L. Remez y A. Tartaglione, Methodologies for estimating abortion incidence and abortion-related morbidity: A review, 2010, pp. 71-97.

48. Singh, S. y A. Bankole. "Estimación de la incidencia del aborto inducido: refutando una crítica a la metodología del Guttmacher Institute", 2012, [https://www.guttmacher.org/media/resources/responseto-methodology-critique-SP.pdf].

49. SIVIGILA. "Panorama de la mortalidad materna en Colombia, 19952000: logros, fracasos, compromisos y retos”, Semana Epidemiológica 37, 2001, [http://www.col.ops-oms.org/sivigila/2001/BOLE37_2001.htm].

50.UN. World abortion policies, [http://www.un.org/en/development/desa/ population/publications/policy/world-abortion-policies-2013.shtml].

51. WHO. "Spontaneous and induced abortion", Report of a WHO Scientific Group", World Health Organization Technical Report Series n. ${ }^{\circ}$ 461, 1970 [http://apps.who.int/iris/bitstream/10665/38211/1/ WHO_TRS_461.pdf].

52.WHO. "The prevention and management of unsafe abortion", Report of a Technical Working Group, 1992, [http://apps.who.int/iris/bitstream/10665/59705/1/WHO_MSM_92.5.pdf].

53. WHO. "Unsafe abortion. Global and regional estimates of the incidence of unsafe abortion and associated mortality in 2000", 2004, [http://apps.who.int/iris/bitstream/10665/42976/1/9241591803.pdf].

54.WHO. Unsafe abortion. Global and regional estimates of the incidence of unsafe abortion and associated mortality in 2008, $6^{\text {th }}$ ed., 2008, [http:// apps.who.int/iris/bitstream/10665/44529/1/9789241501118_eng.pdf].

55. Wilson, K.; S. García y D. Lara. "Misoprostol use and its impact on measuring abortion incidence and morbidity", S. Singh, L. Remez y A. Tartaglione, Methodologies for estimating abortion incidence and abortion-related morbidity: A review, 2010, pp. 191-201.

56. Zamudio, L. et al. "E1 aborto inducido en Colombia. Características demográficas y socioculturales”, Cuadernos del CIDS, Bogotá, Universidad Externado de Colombia, 1999. 